\title{
Caffeine as a tool for investigating the integration of Cdc25 phosphorylation, activity and ubiquitin-dependent degradation in Schizosaccharomyces pombe
}

\author{
John P. Alao ${ }^{1,2^{*}}$ iD and Per Sunnerhagen ${ }^{2}$
}

\begin{abstract}
The evolutionarily conserved Cdc25 phosphatase is an essential protein that removes inhibitory phosphorylation moieties on the mitotic regulator Cdc2. Together with the Wee1 kinase, a negative regulator of Cdc2 activity, Cdc25 is thus a central regulator of cell cycle progression in Schizosaccharomyces pombe. The expression and activity of Cdc25 is dependent on the activity of the Target of Rapamycin Complex 1 (TORC1). TORC1 inhibition leads to the activation of Cdc25 and repression of Wee1, leading to advanced entry into mitosis. Withdrawal of nitrogen leads to rapid Cdc25 degradation via the ubiquitin- dependent degradation pathway by the Pub1 E3- ligase. Caffeine is believed to mediate the override of DNA damage checkpoint signalling, by inhibiting the activity of the ataxia telangiectasia mutated (ATM)/Rad3 homologues. This model remains controversial, as TORC1 appears to be the preferred target of caffeine in vivo. Recent studies suggest that caffeine induces DNA damage checkpoint override by inducing the nuclear accumulation of Cdc25 in S. pombe. Caffeine may thus modulate Cdc25 activity and stability via inhibition of TORC1. A clearer understanding of the mechanisms by which caffeine stabilises $C d c 25$, may provide novel insights into how TORC1 and DNA damage signalling is integrated.
\end{abstract}

Keywords: Schizosaccharomyces pombe, Fission yeast, Ubiquitin, 26S proteasome, Cdc25, Caffeine, Cell cycle, DNA damage checkpoints, Phosphorylation

\section{Background}

The tightly regulated timing of mitosis in S. pombe occurs via the reciprocal activities of Cdc25 and Wee1 on Cdc2 inhibitory phosphorylation. Wee1 negatively regulates Cdc2 by phosphorylation of tyrosine residue 15 (Tyr15), and this is counteracted by the phosphatase activity of Cdc25 [1-3]. Cells must advance or delay mitosis under nutrient stress or genotoxic/environmental stress conditions respectively, several signalling pathways converge on the regulation of the Cdc25- Wee1 dual switch to

\footnotetext{
*Correspondence: j.p.alao@uel.ac.uk

${ }^{1}$ School of Health, Sports and Bioscience, University of East London, Stratford Campus, London E15 4LZ, UK

Full list of author information is available at the end of the article
}

effect accelerated entry into mitosis or a "double- lock" checkpoint mechanism. These pathways include the Target of Rapamycin Complex 1 (TORC1), the DNA damage response (DDR) and the environmental stress response (ESR) pathways [3-7] (Fig. 1).

The methylxanthine caffeine is among the most widely used neuroactive substances in the world [8-11]. Caffeine exerts various effects on cellular and organismal physiology and is known to inhibit several members of the phosphatidylinositol 3 kinase-like kinase (PIKK) family including ataxia telangiectasia mutated (ATM) and ataxia telangiectasia and rad related (ATR) kinase homologue Rad3 and TORC1 in vitro [10, 12-14]. Early studies suggested that caffeine overrides DNA damage checkpoint signalling, by inhibiting Rad3 and its homologues 


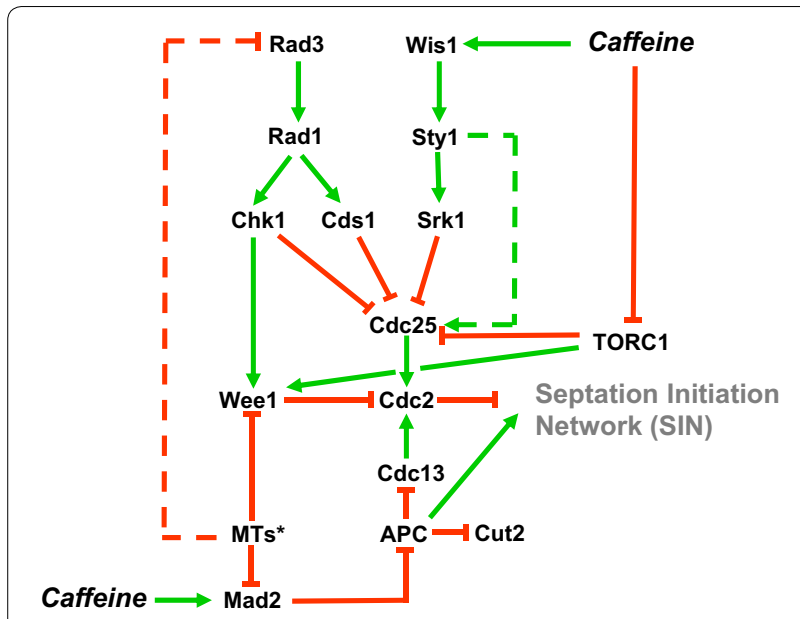

Fig. 1 Effect of caffeine on Cdc25 regulation in S. pombe. Cdc2- $\mathrm{Cdc} 13$ is regulated by $\mathrm{Cdc} 25$ and Wee1. Suppression of Cdc2 activity by the anaphase promoting complex (APC), facilitates mitotic exit and activation of the septation initiation network (SIN). Caffeine was initially thought to inhibit Rad3 activity resulting in DNA damage checkpoint override. More recent studies have identified the TORC1 complex as the major target of caffeine in vivo. TORC1 delays mitosis by negatively regulating $\mathrm{Cdc} 25$ and activating Wee1. TORC1 inhibition advances the timing of mitosis suggesting caffeine can modulate cell cycle progression by inhibiting this complex. Caffeine activates the Sty1 regulated environmental stress response (ESR) pathway, leading to partial Cdc25 inhibition by Srk1. Depending on the degree of activation, Sty 1 can also modulate Cdc25 activity to advance mitosis. The Mad2 spindle checkpoint protein is involved in the regulation of the DNA replication checkpoint. Caffeine's effect on cell cycle progression is partially inhibited by Mad2. *MTs (Microtubules). Green arrows indicate target activation. Red lines indicate inhibitory signalling

but this view remains controversial $[12,15]$. Interestingly, TORC1 appears to be the major cellular target of caffeine in vivo [15-17]. The Tor2-containing TORC1 complex is a negative regulator of $\mathrm{Cdc} 25$ activity that determines the timing of mitosis in response to nutrient availability $[18,19]$. We and others have previously demonstrated that caffeine induces $\mathrm{Cdc} 25$ accumulation in mammalian and $S$. pombe cells $[20,21]$. The mechanisms by which caffeine stabilises Cdc25 in S. pombe remain unclear, but do not result from increased $c d c 25^{+}$mRNA expression. Furthermore, Cdc25 expression was required for caffeine- mediated DNA damage checkpoint override in S. pombe. Intriguingly the effect of caffeine on cell progression under normal growth conditions mimics that of TORC1 inhibition [21]. Caffeine may thus modulate the activity of several pathways that converge on the regulation of Cdc25. In fact, caffeine clearly activates the ESR pathway [21, 22]. One interesting question concerns how, the regulation of Cdc25 activity, phosphorylation and ubiquitin- dependent degradation of Cdc25 activity is integrated [23-25]. Given that cross talk occurs between the TORC1, DDR and ESR pathways [26-28], understanding how caffeine modulates Cdc 25 activity and stability in $S$. pombe may shed further light on how these pathways interact $[4,6,21,29]$. Although the coregulation of Cdc25 and Wee1 is crucial for the proper timing of mitosis or cell cycle arrest and is effected via the same pathways [30]; this review will focus mainly on Cdc25 regulation for simplicity.

\section{Main text \\ Cell cycle dependent regulation of Cdc25 activity, phosphorylation and ubiquitin- dependent degradation by the $26 \mathrm{~S}$ proteasome}

Cdc25 levels oscillate during cell cycle progression in a manner similar to cyclins, rising steadily throughout the cell cycle, before becoming hyper- phosphorylated and degraded during mitosis [1, 2, 23, 31]. Expression of Cdc25 appears to be dependent on TORC1 activity, as nutrient deprivation leads to a rapid loss of expression $[1,2]$. In the absence of a nitrogen source, $c d c 25^{+}$mRNA translation ceases and the protein is rapidly degraded via the ubiquitin- dependent $26 \mathrm{~S}$ proteasome pathway [3234]. Wee1- mediated phosphorylation of Cdc2 tyrosine residues negatively regulates the activity of $\mathrm{Cdc} 2-\mathrm{Cdc} 13$ Maturation Promoting Factor (MPF). Cdc25 removes inhibitory phosphorylation on the $\mathrm{Cdc} 2$, leading to an autocatalytic positive feed- back loop, repression of Wee1 activity and full Cdc25 activation [1,31,35].

The HECT- type ubiquitin ligase Pub1 targets Cdc25 for ubiquitin- dependent $26 \mathrm{~S}$ proteasome degradation in S. pombe. Deletion of $p u b 1^{+}$raises Cdc25 levels and renders cells resistant to Wee1 activity. Furthermore, the cyclic expression pattern of Cdc25 appears deregu-

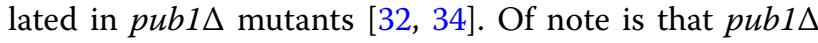
mutants exhibit several phenotypes, suggesting additional Pub1 substrates. Interestingly, Pub1 also controls the ubiquitin- dependent regulation of amino acid uptake potentially linking nutrient absorption to $\mathrm{Cdc} 25$ and cell division via Sty1 and TORC1 [36-38]. The AnaphasePromoting Complex (APC) may also facilitate the degradation of Cdc25 at mitosis [39, 40].

Cdc25 is a highly unstable protein with a relatively short half- life [2, 34]. Cdc25 levels oscillate through the cell cycle, peaking at mitosis and then rapidly decline just prior to cytokinesis [1, 2, 23, 34]. Recent studies by Lucena et al. [23] reveal that Cdc25 in S. pombe becomes highly phosphorylated in G2, becomes dephosphorylated and then hyper- phosphorylated between mitosis and cytokinesis. Cdc25 levels then decline as the cells proceed through mitosis. Phosphorylation of Cdc 25 during normal cell cycle progression is dependent on Cdc2 phosphorylation sites $[23,41]$. The decrease in both phosphorylated and total Cdc25 levels was strongly associated 
with a rise in cyclin Cdc13 levels [23]. Dephosphorylation of Cdc 25 at mitosis is regulated by the protein phosphatase $2 \mathrm{~A}$ and its regulatory subunit Pab1 (PP2A $\left.{ }^{\text {Pab1 }}\right)$. In mutants lacking pab1 $1^{+}, \mathrm{Cdc} 25$ remains hyperphosphorylated throughout the cell cycle and the timing of mitosis exceeds that of wild type cells. The degradation of Cdc25 still occurs in strains expressing mutant isoforms lacking Cdc2 phosphorylation sites, as well as in pab1 $\Delta$ mutants. In addition, the relative abundance of Cdc25 during the cell cycle in pab1 $1 \Delta$ mutants is unaffected [23]. We have also detected a Cdc25 expression negative- feedback loop in S. pombe [21]. Similarly, Clp1 phosphatase activity enhances the rate of Pub1-mediated Cdc25 degradation and timing of mitosis [34, 39, 42]. In clp1 $\Delta$ mutants, Cdc25 remains phosphorylated throughout the cell cycle and the cell cycle is lengthened relative to wild type cells. Levels of Cdc 25 are also elevated relative to wild type cells in $c l p 1 \Delta$ mutants [23, 34]. Clp1 also cooperates with the Pub1 and APC E3- ligases to facilitate the rapid degradation of Cdc25 at mitosis [34, 39, 40, 42]. PP2A ${ }^{\text {Pab1 } 1}$ and Clp1 phosphatase activity and Cdc 25 degradation are thus important for regulating the timing of mitosis. In fact, high Cdc2 activity delays the timing of mitosis in $S$. pombe by inhibiting the septation initiation network (SIN) [34, 39, 40]. Hence, the link between Cdc25 phosphorylation, activity and degradation remains unclear (discussed further below) [24].

Importantly, under normal cell cycle conditions TORC1 inhibits the Greatwall kinase phosphorylates Endosulfine, which is a potent inhibitor of PP2A $\mathrm{A}^{\mathrm{Pab} 1}$ phosphatase activity. When nitrogen is withdrawn or TORC1 is chemically inhibited, PP2 $\mathrm{A}^{\mathrm{Pab} 1}$ is indirectly inhibited, Cdc25 becomes hyperphosphorylated and entry into mitosis in these cells is advanced. This activity also links the Sty1 regulated environmental stress response pathway to TORC 1 and $\mathrm{Cdc} 25$ regulation [43, 44]. Lucena et al. also reported that Cdc25 phosphorylation and dephosphorylation still occur in pab1 $1 \Delta$ mutants [23]. This study did not address however, the role of Srk1dependent Cdc25 phosphorylation during the normal cell cycle (reviewed below). As Srk1- dependent phosphorylation of Cdc25 does not involve the phosphorylation of $\mathrm{Cdc} 2$ consensus sites, sequential and differential phosphorylation or combinations thereof may determine the precise timing of mitosis $[23,25,45]$. TORC 1 thus regulates the timing of mitosis by modulating $\mathrm{PP} 2 \mathrm{~A}^{\mathrm{Pab} 1}$ activity to inhibit Cdc25 and activate Wee1. In contrast, TORC1 inhibition results in Cdc25 activation and the degradation of Wee1 $[18,19,44]$. As PP2A ${ }^{\text {Pab1 }}$ and Clp1 also regulate the phosphorylation, activity and localisation of Wee1, these pathways serve to integrate Cdc25 and Wee1 activity for the proper timing of mitosis [18, 19, 30].

\section{DNA damage checkpoints and Cdc25 inhibition}

Stalled replication during S- phase or DNA strand breaks in G2, activate the Rad3 regulated DNA damage response pathway and respective downstream activation of Cds1 and Chk1 kinases (reviewed in [3, 4]). The Cds1 and Chk1 kinases in turn, phosphorylate key inhibitory serine and threonine residues on $\mathrm{Cdc} 25$. In addition to inhibiting Cdc 25 activity within the nucleus, the phosphorylation of these residues also facilitates binding of the 14-3-3 protein Rad24, nuclear export and sequestration within the cytoplasm [46-49]. Interestingly, Cdc25 levels accumulate in the cytoplasm under conditions of cell cycle arrest following DNA damage. Cdc25 levels also accumulate when cell cycle mutants cease dividing at the restrictive temperature. This "stockpiling" of inactive Cdc25 may facilitate rapid cell cycle re-entry following the completion of DNA damage repair [31]. Later studies indicated that Cdc25 nuclear export is not required for DNA damage checkpoint enforcement, indicating that Cds1 or Chk1 mediated phosphorylation is sufficient to inhibit the activity of the phosphatase $[47,50]$.

Other studies suggest that additional redundant pathways exist, for the regulation of Cdc25 mutants that cannot be phosphorylated [51, 52]. When the 9- 12 major inhibitory phosphorylation sites are mutated $\left.\left(\mathrm{Cdc} 25_{(9 \mathrm{~A}}\right)-\mathrm{GFP}_{\text {int }}, \mathrm{Cdc} 25_{(12 \mathrm{~A})}-\mathrm{GFP}_{\text {int }}\right)$, S. pombe cells are still able to activate an effective DNA damage response. This form of DNA damage checkpoint activation, results from the rapid degradation of these mutant $\mathrm{Cdc} 25$ isoforms and a Mik1 dependent cell cycle arrest [51, 52]. The Cdc $25_{(9 \mathrm{~A})}-\mathrm{GFP}_{\text {int }}$ and $\mathrm{Cdc} 25_{(12 \mathrm{~A})}{ }^{-G F P}$ int expression levels are relatively stable under normal cell cycle conditions, accumulate in the nucleus to a greater extent than the wild type Cdc25-GFP int but have a slightly shorter half- life.

Enforced nuclear localisation of Cdc25 (Cdc25- NLS$\left.\mathrm{GFP}_{\text {int }}\right)$ does not affect replication checkpoint activation and stockpiling of the phosphatase occurs as with the wild type isoform. The levels of Cdc25- NLS- GFP int are also relatively higher, than in wild type Cdc25- GFP. In contrast, $\mathrm{Cdc} 25_{(9 \mathrm{~A})}-\mathrm{NLS}-\mathrm{GFP}_{\text {int }}$ is degraded when the replication checkpoint is activated. Cdc $25_{(9 A)}$ - NLS$\mathrm{GFP}_{\text {int }}$ also appears to be relatively unstable compared to Cdc25- NLS- GFP ${ }_{\text {int }}$, suggesting Cdc25 phosphorylation prevents degradation during the normal cell cycle $[51,52]$. These observations indicate that Cdc25 degradation occurs in the nucleus following stalled replication or DNA damage. They also suggest that activation of the replication or DNA damage checkpoints, induces an increase in the rate of non- phosphorylated Cdc25 degradation. In this regard, it is important to note that Cut8 localises the $26 \mathrm{~S}$ proteasome to the nucleus, accumulates following DNA damage and is required for 
DNA repair. However, mutants lacking cut $^{+}$are checkpoint proficient [53]. As wild type Cdc25 degradation is not required for replication stress or DNA damageinduced cell cycle arrest, it would be interesting to study the impact of a cut8 deletion on Cdc25 $5_{(9 \mathrm{~A})}-\mathrm{GFP}$ int and Cdc25 $5_{(12 \mathrm{~A})}-\mathrm{GFP}_{\text {int }}$ degradation. Cds1 or Chk1- mediated phosphorylation of the major inhibitory phosphorylation sites is thus sufficient to prevent degradation by the $26 \mathrm{~S}$ proteasome. Other lines of evidence suggest, that the Rad3 regulated checkpoint pathways regulate $\mathrm{Cdc} 25$ expression and stability even under normal growth conditions. Deletion of $\mathrm{rad}^{+}$or $c d s 1^{+}$suppressed $c d c 25^{+}$ mRNA expression but induced the accumulation the Cdc25 protein. Unlike wild type cells, $\operatorname{rad} 3 \Delta$ mutants continue to express Cdc25 even in stationary phase [21]. Similarly, the rate of degradation of $\mathrm{Cdc} 25_{(9 \mathrm{~A})}-\mathrm{GFP}_{\text {int }}$ and Cdc25 $5_{(12 \mathrm{~A})}$-GFP $_{\text {int }}$ mutant protein is delayed in a $c d s 1 \Delta$ background [52]. Rad3 may thus regulate Cdc 25 stability in a Cds1- dependent manner even under normal growth conditions. Cds1 also accumulates in response to TORC1 inhibition following glucose withdrawal, providing a further link between TORC and DNA damage checkpoint signalling [54]. While the Pub1 E3- ligase targets Cdc25 to the $26 \mathrm{~S}$ proteasome for degradation, deletion of $p u b 1^{+}$ did not prevent the degradation of $\mathrm{Cdc} 25_{(9 \mathrm{~A})}-\mathrm{GFP}_{\text {int }}$ mutant protein in the presence of hydroxyurea [52]. Furthermore, $p u b 1 \Delta$ mutants have elevated Cdc 25 levels, fail to adequately degrade the phosphatase at mitosis and are sensitive to genotoxic agents [23, 32, 55]. Interestingly, mutants also display sensitivity to caffeine ([56], Alao and Sunnerhagen, unpublished results). It is thus possible that the APC mediates the degradation of Cdc25 isoforms lacking major inhibitory phosphorylation sites, following the activation of the replication or DNA damage checkpoints $[39,40,52]$. Indeed, APC mediated degradation of mitotic cyclins and regulators is required for proper exit from mitosis and progression through cytokinesis [39]. Clp1 is also required for full activation of Cds1 in response to replication stress [57]. Interactions between the replication checkpoint and spindle checkpoint pathways also contribute to the enforcement of cell cycle arrest under genotoxic conditions. These interactions may also contribute to the regulation of $\mathrm{Cdc} 25$ stability, via differential combinations of positive (Cdk1, Plo1 mediated) and negative (Cds1, Chk1, Srk1 mediated) phosphorylation of serine/threonine residues [3, 21, $29,40,58]$ in DNA damage checkpoints and Cdc25 inhibition section [59-65].

In $\mathrm{Cdc} 25_{(9 \mathrm{~A})}-\mathrm{GFP}_{\text {int }}$ mutants Mik1 is required for effective maintenance of the replication checkpoint $[51,52]$. Thus, while Rad24 binding slightly enhances Cdc25 stability under normal growth conditions, it prevents the degradation of the phosphatase when the DNA damage or replication checkpoint pathways are activated. The existence of these redundant mechanisms suggests that even modest Cdc 25 activity during DNA damage checkpoint activation can contribute to inappropriate progression through mitosis $[21,51,52]$.

Genomic studies have also revealed a role for the DNA damage response pathway, in mediating resistance to caffeine. Mutants with $\mathrm{rad} 3 \Delta, \mathrm{rad} 51 \Delta$, or $\mathrm{rad} 54 \Delta$ mutations also show sensitivity when grown on solid media in the presence of caffeine [22]. Caffeine may thus induce DNA damage, but the underlying mechanisms remain unclear. It is interesting to note however, that these findings hint at caffeine- induced DNA damage and Rad3 activation in S. pombe. Caffeine also appears to accelerate the timing of mitosis under genotoxic conditions, rather than delaying cell cycle progression. Together, these observations provide additional evidence that $\operatorname{Rad} 3$ is in fact not a target of caffeine in this organism [21].

\section{Effect of caffeine on Cdc25 expression and stability}

Caffeine can inhibit several members of the PIKK family, and inhibition of Rad3 and its homologues ATM and ATR was thought to be the mechanism underlying checkpoint override [10, 12-14]. This paradigm has proved controversial, as checkpoint override by caffeine can occur in the absence of ATM, ATR or Rad3 inhibition $[15,21,66]$. It has also become apparent, that TORC1 and not ATM homologues are the preferred target of caffeine in vivo [15-17]. TORC1 regulates the timing of cell division in response to nutrient availability via the $S$. pombe Greatwall kinase homologue Ppk18 [18, 67]. Inhibition of TORC1 activity activates Cdc 25 , induces Wee1 degradation and advances cells into mitosis. The exposure of $S$. pombe cells to caffeine advances mitosis in a manner that resembles TORC1 inhibition [21]. Caffeine also moderately activates the Sty1- regulated ESR pathway [21, 22]. Modest Sty1 activation can drive cells into mitosis in a manner dependent on Plo1 and Cdc25 [6, 43]. Activation of Sty1 has been shown to induce Cdc25 stabilisation, presumably as a consequence of Srk1- mediated phosphorylation, Rad24 binding and sequestration within the cytoplasm [25]. Caffeine may thus modulate cell cycle progression by partially inhibiting TORC1, moderately activating Sty 1 or otherwise modulating Cdc 25 activity to advance mitosis. In fact, Cdc 25 expression was necessary for caffeine- mediated DNA damage checkpoint override in our studies [21]. Previous studies have shown that caffeine induces the accumulation of Cdc25B in mammalian cells [20]. We have similarly demonstrated that caffeine induces the accumulation of Cdc25 in S. pombe under normal cell cycle conditions as well as under environmental stress or genotoxic conditions. This effect on Cdc25 occurs at the post-translational level since caffeine 
suppresses $c d c 25^{+}$mRNA expression. Interestingly, rad3 $3 \Delta$ and $c d s 1 \Delta$ deletions also stabilised Cdc25 protein levels while supressing its mRNA expression. Furthermore, caffeine is more effective at advancing mitosis in $\operatorname{rad} 3 \Delta$ and $c d s 1 \Delta$ mutants relative to wild type cells [21]. We also noted that DNA damage checkpoint mutants do not just fail to arrest cell division but are accelerated into mitosis following DNA damage. This change in cell cycle kinetics resembles the effect of caffeine on cells exposed to genotoxic agents [21, 29, 68, 69]. Caffeine thus mimics the loss of DNA damage checkpoint signalling in $S$. pombe, without inhibiting Rad3 activity [21]. This effect of caffeine also mimics that of the Tor2 inhibitors rapamycin and torin1 on cell cycle progression in S. pombe [44]. Mutants lacking functional Clp1 or Srk1 that normally negatively regulate $\mathrm{Cdc} 25$ are more sensitive to caffeine mediated DNA damage checkpoint override than wild type cells. The phosphorylation of Cdc25 is therefore not required for the stabilising effect of caffeine on the phosphatase but influences its effect on cell cycle progression [21].

Caffeine inhibits the degradation of Cdc25 mutants $\left(\mathrm{Cdc} 25_{(9 \mathrm{~A})}-\mathrm{GFP}_{\text {int }}\right.$ and $\left.\mathrm{Cdc} 25_{(12 \mathrm{~A})}-\mathrm{GFP}_{\text {int }}\right)$ lacking the major inhibitory phosphorylation sites [21, 51, 52]. In contrast to the stockpiling of wild type Cdc 25 when cells are arrested, the $\mathrm{Cdc} 25_{(9 \mathrm{~A})}-\mathrm{GFP}_{\text {int }}$ and $\mathrm{Cdc} 25_{(12 \mathrm{~A})}-\mathrm{GFP}_{\text {int }}$ mutants are degraded in the presence of genotoxic agents. Redundant mechanisms thus exist, to clear excess non- phosphorylated Cdc25 from the nucleus when DNA damage checkpoint signalling is activated [51, 52]. Caffeine clearly stabilised these mutants in the presence of genotoxic agents [21]. As $\operatorname{Cdc} 25_{(9 \mathrm{~A})}-\mathrm{GFP}_{\text {int }}$ and $\mathrm{Cdc} 25_{(12 \mathrm{~A})}-\mathrm{GFP}_{\text {int }}$ are relatively stable under normal cell cycle conditions, caffeine must inhibit a pathway that targets non- phosphorylated Cdc25 for ubiquitindependent $26 \mathrm{~S}$ proteasomal degradation under genotoxic conditions. The ability of caffeine to override checkpoint signalling in cells expressing these mutants, is also enhanced relative to the wild type protein $[21,51,52]$.

The rapid degradation of Cdc 25 isoforms that cannot be phosphorylated $\left(\mathrm{Cdc} 25_{(9 \mathrm{~A})}-\mathrm{GFP}_{\text {int }}\right.$ and $\left.\mathrm{Cdc} 25_{(12 \mathrm{~A})}-\mathrm{GFP}_{\text {int }}\right)$ [51] following genotoxic insults, hints at an increase in $26 \mathrm{~S}$ proteasome mediated protein degradation. This redundant mechanism clears $\mathrm{Cdc} 25$ that is unphosphorylated from the nucleus $[51,52]$. These studies also demonstrated that $\mathrm{Cdc} 25$ protection from degradation occurs via Chk1 and Cds1 inhibitory phosphorylation. As these isoforms are relatively stable under normal cell cycle conditions, genotoxic conditions must somehow enhance the targeting of unphosphorylated Cdc25 to the $26 \mathrm{~S}$ proteasome $[51,52]$. Caffeine thus suppresses Cdc25 degradation independently of Cds1, Chk1 and Srk1-mediated phosphorylation [21]. In fact, exposure to $0.6 \mathrm{M} \mathrm{KCl}$ induced the degradation of $\mathrm{Cdc} 25_{(9 \mathrm{~A})}-\mathrm{GFP}_{\text {int }}$ in a manner similar to what was observed with genotoxic agents (Alao and Sunnerhagen, unpublished results).

\section{Mechanisms underlying caffeine- induced Cdc25 stabilisation}

By what mechanism(s) could caffeine affect the rate of Cdc25 degradation via the $26 \mathrm{~S}$ proteasome? Caffeine has been reported to induce the ubiquitin- dependent degradation of certain proteins in mammalian cells [70]. The rapid degradation of Cdc25 isoforms that cannot be phosphorylated under genotoxic conditions, hints at the activation (or increased activity) of a ubiquitin- dependent degradation pathway. Alternatively, a general increase in the overall rate of ubiquitin- dependent degradation may occur under these conditions. Clearly further studies on the regulation of Pub1 (the E3- ligase targeting Cdc25) activity under normal and genotoxic conditions, in the presence and absence of caffeine are warranted. Such studies may also provide novel insights into the regulation of $\mathrm{Cdc} 25$ stability in S. pombe. Similarly, Cut8 is required to localise the $26 \mathrm{~S}$ proteasome to the nucleus and plays an important role in DNA damage repair. Cut8 accumulates in response to DNA damage but is not required for checkpoint activation [53, 71]. The accumulation of Cut8 in the presence of genotoxic agents suggests a possible increase in the levels of ubiquitin- dependent protein degradation and could also drive progression through mitosis. Inhibiting Cut8 accumulation could be one possible mechanism, whereby caffeine attenuates the ubiquitin- degradation of nuclear Cdc25 (Alao and Sunnerhagen, unpublished results). Interestingly, cut $8 \Delta$ mutants also display sensitivity to caffeine [22]. These observations suggest that caffeine is itself a DNA damaging agent $[22,53]$ and may complicate studies on the effect of the drug on the DNA damage response pathway. Nevertheless, the ability of caffeine to override checkpoint signalling and drive cells trough mitosis appears to underlie its chemo- and radio- sensitising effects [9]. Lastly, studies on the effect of caffeine- mediated TORC1 inhibition in the context of mitotic progression are also potentially important. TORC1 mediates the timing of mitosis, by co-ordinating the phosphorylation, activity and expression levels of Cdc25 and Wee1 [18, 23, 44]. The effect of caffeine on cell cycle progression resembles that of more typical TORC1 inhibitors by accelerating the timing of mitosis in $S$. pombe [21, 44]. Caffeine could thus advance the timing of mitosis, by indirectly increasing Cdc25 activity while inhibiting the activity of Wee1. Comparing the effects of TORC1 inhibitors on checkpoint activation with those of caffeine would be interesting. New 
antibodies that detect hyperphosphorylated Cdc25 and Wee1 have recently been reported. Studies on the effect of caffeine on cell cycle progression in various genetic backgrounds (e.g. mutants of the TORC1 signalling pathway such as pab1 $\Delta$ ) using these tools would also be useful [23].

\section{Conclusion}

Despite more than two decades of research, the precise mechanisms whereby caffeine overrides checkpoint signalling remain unclear $[9,10,17,21,66]$. The more recent findings that TORC1 and not Rad3 appears to be the major target of caffeine in vivo, is particularly relevant in this regard [15]. It is thus likely that caffeine override DNA damage checkpoint signalling independently of Rad3 inhibition. Modulation of TORC activity by caffeine could account for its effects on cell cycle progression [17, 44] (Fig. 1). Furthermore, caffeine also targets other pathways, at least some of which interact with each other [21, 29]. Clearly, understanding how caffeine suppresses the degradation of Cdc25 in S. pombe is of central importance. Studies of this nature may shed light not only on the molecular pharmacology of caffeine, but also on how signalling pathway crosstalk impacts on cell cycle regulation. With the new insights and tools available, we can look forward to many more years of exciting research in this area.

\section{Abbreviations \\ APC: AnaphasePromoting Complex; ATM: Ataxia telangiectasia mutated; ATR : Ataxia and rad related (ATR) kinase homologue Rad3; DDR: DNA damage response; ESR: Environmental stress response; PIKK: Phosphatidylinositol 3 kinase-like kinase; TORC1: Target of rapamycin complex 1.}

\section{Acknowledgements}

Not applicable.

\section{Authors' contributions}

JPA and PS conceived of the manuscript. JPA wrote the manuscript. JPA and PS read and edited the manuscript. Both authors read and approved the final manuscript.

\section{Funding}

This work was financially supported by grants from the Swedish Cancer Fund (19-0133) and the Carl Tryggers foundation (15:13).

\section{Availability of data and materials}

Not applicable.

Ethics approval and consent to participate

Not applicable.

\section{Consent for publication}

Not applicable.

\section{Competing interests}

The authors declare there is no competing interest.

\section{Author details}

${ }^{1}$ School of Health, Sports and Bioscience, University of East London, Stratford Campus, London E15 4LZ, UK. ${ }^{2}$ Department of Chemistry and Molecular Biology, University of Gothenburg, Box 462, Gothenburg SE- 405 30, Sweden.

Received: 31 January 2020 Accepted: 8 June 2020

Published online: 29 June 2020

\section{References}

1. Moreno S, Nurse P, Russell P. Regulation of mitosis by cyclic accumulation of p80 ${ }^{\mathrm{cdc} 25}$ mitotic inducer in fission yeast. Nature. 1990;344:549-52.

2. Ducommun B, Draetta G, Young P, Beach D. Fission yeast cdc 25 is a cellcycle regulated protein. Biochem Biophys Res Commun. 1990;167:301-9.

3. Karlsson-Rosenthal C, Millar JB. Cdc25: mechanisms of checkpoint inhibition and recovery. Trends Cell Biol. 2006;16:285-92.

4. Alao JP, Sunnerhagen P. Rad3 and Sty1 function in Schizosaccharomyes pombe: an integrated response to DNA damage and environmental stress? Mol Microbiol. 2008;68:246-54.

5. Raleigh JM, O'Connell MJ. The G(2) DNA damage checkpoint targets both Wee1 and Cdc25. J Cell Sci. 2000;113:1727-36.

6. Petersen J. TOR signalling regulates mitotic commitment through stressactivated MAPK and Polo kinase in response to nutrient stress. Biochem Soc Transact. 2009;37:273-7.

7. Yanagida M, Ikai N, Shimanuki M, Sajiki K. Nutrient limitations alter cell division control and chromosome segregation through growthrelated kinases and phosphatases. Philos Trans R Soc Lond B Biol Sci. 2011;366:3508-20.

8. Butt MS, Sultan MT. Coffee and its consumption: benefits and risks. Crit Rev Food Sci Nutrition. 2011;51:363-73.

9. Moser BA, Brondello JM, Baber-Furnari B, Russell P. Mechanism of caffeine-induced checkpoint override in fission yeast. Mol Cell Biol. 2000;20:4288-94.

10. Bode AM, Dong Z. The enigmatic effects of caffeine in cell cycle and cancer. Cancer Lett. 2007;247:26-39.

11. Takahashi K, Yanai S, Shimokado K, Ishigami A. Coffee consumption in aged mice increases energy production and decreases hepatic mTOR levels. Nutrition. 2017;38:1-8.

12. Lovejoy CA, Cortez D. Common mechanisms of PIKK regulation. DNA Repair. 2009:8:1004-8.

13. Humphrey T. DNA damage and cell cycle control in Schizosaccharomyces pombe. Mutat Res. 2000;451:211-26.

14. Gibbs BF, Goncalves Silva I, Prokhorov A, Abooali M, Yasinska IM, CaselyHayford MA, Berger SM, Fasler-Kan E, Sumbayev W. Caffeine affects the biological responses of human hematopoietic cells of myeloid lineage via downregulation of the mTOR pathway and xanthine oxidase activity. Oncotarget. 2015;6:28678-92.

15. Wanke V, Cameroni E, Uotila A, Piccolis M, Urban J, Loewith R, De Virgilio C. Caffeine extends yeast lifespan by targeting TORC1. Mol Microbiol. 2008;69:277-85.

16. Reinke A, Chen JC, Aronova S, Powers T. Caffeine targets TOR complex I and provides evidence for a regulatory link between the FRB and kinase domains of Tor1p. J Biol Chem. 2006;281:31616-26.

17. Rallis C, Codlin S, Bähler J. TORC1 signaling inhibition by rapamycin and caffeine affect lifespan, global gene expression, and cell proliferation of fission yeast. Aging Cell. 2013;12:563-73.

18. Perez-Hidalgo L, Moreno S. Coupling TOR to the cell cycle by the Greatwall-Endosulfine-PP2A-B55 pathway. Biomolecules. 2017;7(3):59.

19. Martin R, Lopez-Aviles S. Express yourself: how PP2A-B55(Pab1) helps TORC1 talk to TORC2. Curr Genet. 2018;64:43-51.

20. Varmeh S, Manfredi JJ. Inappropriate activation of cyclin-dependent kinases by the phosphatase $\mathrm{Cdc} 25 \mathrm{~b}$ results in premature mitotic entry and triggers a p53-dependent checkpoint. J Biol Chem. 2009;284:9475-88.

21. Alao JP, Johansson-Sjölander J, Baar J, Özbaki-Yagan N, Kakoschky B, Sunnerhagen P. Caffeine stabilizes Cdc25 independently of Rad3 in Schizosaccharomyces pombe contributing to checkpoint override. Mol Microbiol. 2014;92:777-96. 
22. Calvo IA, Gabrielli N, Iglesias-Baena I, Garcia-Santamarina S, Hoe KL, Kim DU, Sanso M, Zuin A, Perez P, Ayte J, et al. Genome-wide screen of genes required for caffeine tolerance in fission yeast. PLoS ONE. 2009;4:e6619.

23. Lucena R, Alcaide-Gavilan M, Anastasia SD, Kellogg DR. Wee1 and Cdc25 are controlled by conserved PP2A-dependent mechanisms in fission yeast. Cell Cycle. 2017;16:428-35.

24. Moseley JB. Wee1 and Cdc25: tools, pathways, mechanisms, questions. Cell Cycle. 2017;16:599-600.

25. López-Avilés S, Grande M, González M, Helgesen AL, Alemany V, Sanchez-Piris M, Bachs O, Millar JB, Aligue R. Inactivation of the Cdc25 phosphatase by the stress-activated Srk1 kinase in fission yeast. Mol Cell. 2005;17:49-59.

26. Ma Y, Vassetzky Y, Dokudovskaya S. mTORC1 pathway in DNA damage response. Biochim Biophys Acta Mol Cell Res. 2018;1865:1293-311.

27. Ueda S, Ozaki R, Kaneko A, Akizuki R, Katsuta H, Miura A, Matsuura A, Ushimaru T. TORC1, Tel1/Mec1, and Mpk1 regulate autophagy induction after DNA damage in budding yeast. Cell Signal. 2019;62:109344.

28. Miyamoto I, Ozaki R, Yamaguchi K, Yamamoto K, Kaneko A, Ushimaru T. TORC1 regulates the DNA damage checkpoint via checkpoint protein levels. Biochem Biophys Res Commun. 2019;510:629-35.

29. Alao JP, Huis in't Veld PJ, Buhse F, Sunnerhagen P. Hyperosmosis enhances radiation and hydroxyurea resistance of $S$. pombe checkpoint mutants through the spindle checkpoint and delayed cytokinesis. Mol Microbiol. 2010;77:143-57.

30. de Gooijer MC, van den Top A, Bockaj I, Beijnen JH, Wurdinger T, van Tellingen O. The G2 checkpoint-a node-based molecular switch. FEBS Open Bio. 2017;7:439-55

31. Kovelman R, Russell P. Stockpiling of cdc25 during a DNA replication checkpoint arrest in Schizosaccharomyces pombe. Mol Cell Biol. 1996;16:86-93.

32. Nefsky B, Beach D. Pub1 acts as an E6-AP-like protein ubiquitin ligase in the degradation of cdc25. EMBO J. 1996;15:1301-12.

33. Tamai KK, Shimoda C. The novel HECT-type ubiquitin-protein ligase Pub2p shares partially overlapping function with Pub1p in Schizosaccharomyces pombe. J Cell Sci. 2002;115:1847-57.

34. Esteban V, Sacristan M, Andres S, Bueno A. The Flp1/Clp1 phosphatase cooperates with HECT-type Pub1/2 protein-ubiquitin ligases in Schizosaccharomyces pombe. Cell Cycle. 2008;7:1269-76.

35. Hoffmann I, Clarke PR, Marcote MJ, Karsenti E, Draetta G. Phosphorylation and activation of human $\mathrm{cdc} 25-\mathrm{C}$ by $\mathrm{cdc} 2 \mathrm{Cyclin}-\mathrm{B}$ and its involvement in the self-amplification of MPF at mitosis. EMBO J. 1993;12:53-63.

36. Karagiannis J, Saleki R, Young PG. The pub1 E3 ubiquitin ligase negatively regulates leucine uptake in response to $\mathrm{NH}_{4}{ }^{+}$in fission yeast. Curr Genet. 1999;35:593-601.

37. Nakase Y, Nakase M, Kashiwazaki J, Murai T, Otsubo Y, Mabuchi I, Yamamoto M, Takegawa K, Matsumoto T. The fission yeast beta-arrestin-like protein Any1 is involved in TSC-Rheb signaling and the regulation of amino acid transporters. J Cell Sci. 2013;126:3972-81.

38. Nakashima A, Kamada S, Tamanoi F, Kikkawa U. Fission yeast arrestinrelated trafficking adaptor, Arn1/Any1, is ubiquitinated by Pub1 E3 ligase and regulates endocytosis of Cat1 amino acid transporter. Biol Open. 2014;3:542-52

39. Wolfe BA, Gould KL. Fission yeast Clp1p phosphatase affects G2/M transition and mitotic exit through Cdc25p inactivation. EMBO J. 2004;23:919-29.

40. Wolfe BA, Gould KL. Inactivating Cdc25, mitotic style. Cell Cycle. 2004:3:601-3

41. Lu LX, Domingo-Sananes MR, Huzarska M, Novak B, Gould KL. Multisite phosphoregulation of $\mathrm{Cdc} 25$ activity refines the mitotic entrance and exit switches. Proc Natl Acad Sci USA. 2012;109:9899-904.

42. Trautmann S, Wolfe BA, Jorgensen $P$, Tyers M, Gould KL, McCollum D. Fission yeast Clp1p phosphatase regulates $\mathrm{G} 2 / \mathrm{M}$ transition and coordination of cytokinesis with cell cycle progression. Curr Biol. 2001;11:931-40.

43. Hartmuth S, Petersen J. Fission yeast Tor 1 functions as part of TORC1 to control mitotic entry through the stress MAPK pathway following nutrient stress. J Cell Sci. 2009:122:1737-46.

44. Atkin J, Halova L, Ferguson J, Hitchin JR, Lichawska-Cieslar A, Jordan AM, Pines J, Wellbrock C, Petersen J. Torin 1-mediated TOR kinase inhibition reduces Wee1 levels and advances mitotic commitment in fission yeast and HeLa cells. J Cell Sci. 2014;127:1346-56.

45. López-Avilés S, Lambea E, Moldón A, Grande M, Fajardo A, RodríguezGabriel MA, Hidalgo E, Aligue R. Activation of Srk1 by the MAP kinase Sty $1 /$ Spc1 precedes its dissociation from the kinase and signals its degradation. Mol Biol Cell. 2008;19:1670-9.

46. Furnari B, Rhind N, Russell P. Cdc 25 mitotic inducer targeted by Chk1 DNA damage checkpoint kinase. Science. 1997;277:1495-7.

47. Furnari B, Blasina A, Boddy MN, McGowan CH, Russell P. Cdc25 inhibited in vivo and in vitro by checkpoint kinases Cds1 and Chk1. Mol Biol Cell. 1999;10:833-45.

48. Lopez-Girona A, Furnari B, Mondesert O, Russell P. Nuclear localization of $\mathrm{Cdc} 25$ is regulated by DNA damage and a 14-3-3 protein. Nature. 1999;397:172-5.

49. Zeng Y, Forbes KC, Wu Z, Moreno S, Piwnica-Worms H, Enoch T. Replication checkpoint requires phosphorylation of the phosphatase $\mathrm{Cdc} 25$ by Cds1 or Chk1. Nature. 1998;395:507-10.

50. Lopez-Girona A, Kanoh J, Russell P. Nuclear exclusion of Cdc25 is not required for the DNA damage checkpoint in fission yeast. Curr Biol. 2001;11:50-4.

51. Frazer $C$, Young PG. Redundant mechanisms prevent mitotic entry following replication arrest in the absence of Cdc25 hyper-phosphorylation in fission yeast. PLoS ONE. 2011;6:e21348.

52. Frazer $C$, Young PG. Carboxy-terminal phosphorylation sites in Cdc25 contribute to enforcement of the DNA damage and replication checkpoints in fission yeast. Curr Genet. 2012;58:217-34.

53. Kearsey SE, Stevenson AL, Toda T, Wang SW. Fission yeast Cut8 is required for the repair of DNA double-strand breaks, ribosomal DNA maintenance, and cell survival in the absence of Rqh1 helicase. Mol Cell Biol. 2007;27:1558-67.

54. Fletcher J, Griffiths L, Caspari T. Nutrient limitation inactivates Mrc1to-Cds 1 checkpoint signalling in Schizosaccharomyces pombe. Cells. 2018;7(2):15.

55. Han TX, Xu XY, Zhang MJ, Peng X, Du LL. Global fitness profiling of fission yeast deletion strains by barcode sequencing. Genome Biol. 2010;11:R60.

56. Rallis C, Townsend S, Bähler J. Genetic interactions and functional analyses of the fission yeast gsk3 and amk2 single and double mutants defective in TORC1-dependent processes. Sci Rep. 2017;7:44257.

57. Diaz-Cuervo H, Bueno A. Cds1 Controls the release of Cdc14-like phosphatase Flp1 from the nucleolus to drive full activation of the checkpoint response to replication stress in fission yeast. Mol Biol Cell. 2008;19:2488-99.

58. Sugimoto I, Murakami H, Tonami Y, Moriyama A, Nakanishi M. DNA replication checkpoint control mediated by the spindle checkpoint protein Mad2p in fission yeast. J Biol Chem. 2004;279:47372-8.

59. Asp E, Sunnerhagen P. Mkp1 and Mkp2, two MAPKAP-kinase homologues in Schizosaccharomyces pombe, interact with the MAP kinase Sty1. Mol Genet Genomics. 2003;268:585-97.

60. Degols G, Shiozaki K, Russell P. Activation and regulation of the Spc1 stress-activated protein kinase in Schizosaccharomyces pombe. Mol Cell Biol. 1996;16:2870-7.

61. Smith DA, Toone WM, Chen D, Bähler J, Jones N, Morgan BA, Quinn J. The Srk1 protein kinase is a target for the Sty 1 stress-activated MAPK in fission yeast. J Biol Chem. 2002;277:33411-21.

62. Paul M, Ghosal A, Bandyopadhyay S, Selvam U, Rai N, Sundaram G. The fission yeast MAPK Spc1 senses perturbations in Cdc25 and Wee 1 activities and targets Rad24 to restore this balance. Yeast. 2018;35:261-71.

63. Millar JB, Buck V, Wilkinson MG. Pyp1 and Pyp2 PTPases dephosphorylate an osmosensing MAP kinase controlling cell size at division in fission yeast. Genes Dev. 1995;9:2117-30

64. Shiozaki K, Russell P. Cell-cycle control linked to extracellular environment by MAP kinase pathway in fission yeast. Nature. 1995:378:739-43.

65. Degols G, Russell P. Discrete roles of the Spc1 kinase and the Atf1 transcription factor in the UV response of Schizosaccharomyces pombe. Mol Cell Biol. 1997; 17:3356-63.

66. Cortez D. Caffeine inhibits checkpoint responses without inhibiting the ataxia-telangiectasia-mutated (ATM) and ATM- and Rad3-related (ATR) protein kinases. J Biol Chem. 2003;278:37139-45. 
67. Otsubo Y, Nakashima A, Yamamoto M, Yamashita A. TORC1-dependent phosphorylation targets in fission yeast. Biomolecules. 2017;7(3):50.

68. Alao JP, Sunnerhagen P. The ATM and ATR inhibitors CGK733 and caffeine suppress cyclin D1 levels and inhibit cell proliferation. Radiation Oncol. 2009:4:51-7.

69. Kanter-Smoler G, Knudsen KE, Jimenez G, Sunnerhagen P, Subramani S. Separation of phenotypes in mutant alleles of the Schizosaccharomyces pombe cell cycle checkpoint gene rad $1^{+}$. Mol Biol Cell. 1995;6:1793-805.

70. Gressner OA. About coffee, cappuccino and connective tissue growth factor-Or how to protect your liver!? Environm Toxicol Pharmacol. 2009;28:1-10.
71. Takeda K, Yanagida M. Regulation of nuclear proteasome by Rhp6/Ubc2 through ubiquitination and destruction of the sensor and anchor Cut8. Cell. 2005;122:393-405.

\section{Publisher's Note}

Springer Nature remains neutral with regard to jurisdictional claims in published maps and institutional affiliations.
Ready to submit your research? Choose BMC and benefit from:

- fast, convenient online submission

- thorough peer review by experienced researchers in your field

- rapid publication on acceptance

- support for research data, including large and complex data types

- gold Open Access which fosters wider collaboration and increased citations

- maximum visibility for your research: over $100 \mathrm{M}$ website views per year

At BMC, research is always in progress.

Learn more biomedcentral.com/submissions 